Check for updates

Cite this: DOI: $10.1039 / \mathrm{c} 8 \mathrm{mh} 01510 \mathrm{~d}$

Received 26th November 2018, Accepted 13th December 2018

DOI: 10.1039/c8mh01510d

rsc.li/materials-horizons

\section{Temperature-responsive nanomagnetic logic gates for cellular hyperthermia $\dagger$}

\author{
Rui Oliveira-Silva, ${ }^{a b}$ Rute A. Pereira, ${ }^{a}$ Fábio M. Silva, ${ }^{a}$ Vitor M. Gaspar, \\ Alfonso Ibarra, ${ }^{d}$ Ángel Millán, (D) ${ }^{e}$ Filipa L. Sousa, ${ }^{c}$ João F. Mano (D) ${ }^{c}$ and \\ Nuno J. O. Silva (iD *a
}

While a continuous monitoring of temperature at the micro- and nano-scales is clearly of interest in many contexts, in many others a yes or no answer to the question "did the system locally exceed a certain temperature threshold?" can be more accurate and useful. This is the case of hard-to-detect events, such as those where temperature fluctuations above a defined threshold are shorter than the typical integration time of micro/nanothermometers and systems where fluctuations are rare events in a wide time frame. Herein we present the synthesis of iron selenide magnetic nanoplatelets and their use as non-volatile logic gates recording the near infrared (NIR) dose that triggers a temperature increase above a critical temperature around $42{ }^{\circ} \mathrm{C}$ in prostate cancer cell cultures. This use is based on the bistable behavior shown by the nanoplatelets below a magnetic phase transition at a tunable temperature $T_{\mathrm{C}}$ and on their photothermal response under NIR light. The obtained results indicate that the synthesized nanomagnets may be employed in the future as both local heaters and temperature monitoring tools in a wide range of contexts involving systems which, as cells, are temperature-sensitive around the tunable $T_{\mathrm{C}}$.

Temperature control is a key aspect in many areas of daily life, where devices, food, organisms and molecules are designed to function and preserve their properties within well-defined temperature limits. Devices that can signal a temperature exceeding these limits are vital in all these areas. In other contexts, the crossing of temperature limits is used intentionally, as in the case

\footnotetext{
${ }^{a}$ Departamento de Física and CICECO, Aveiro Institute of Materials, Universidade de Aveiro, 3810-193 Aveiro, Portugal. E-mail: nunojoao@ua.pt

${ }^{b}$ Univ. Lisbon, Inst. Super. Tecn., Inst. Bioengn. \& Biosci., Dept. Bioengn., Ave Rovisco Pais, P-1049001 Lisbon, Portugal

${ }^{c}$ Departamento de Quimica and CICECO, Aveiro Institute of Materials, Universidade de Aveiro, 3810-193 Aveiro, Portugal

${ }^{d}$ LMA, Instituto de Nanociencia de Aragón, Universidad de Zaragoza, Spain

${ }^{e}$ Instituto de Ciencia de Materiales de Aragón, CSIC - Universidad de Zaragoza, Departamento de Física de la Materia Condensada, Facultad de Ciencias, 50009 Zaragoza, Spain

$\dagger$ Electronic supplementary information (ESI) available: Detailed experimental description, temperature distribution simulations, additional TEM images, XRD, XPS, magnetic, DLS and TG data (PDF). See DOI: 10.1039/c8mh01510d
}

\begin{abstract}
Conceptual insights
Gaining insight into temperature at the micro- and nano-scales is currently addressed using nanothermometers based on the well-defined temperature dependence of a given property (e.g. luminescence, spin transitions). This approach is challenged when the temperature range of interest and the timescale are short, such as in cellular hyperthermia, since space, time and temperature resolutions are entangled. Here we propose the use of nanosized logic gates recording temperature overshoots for such a demanding application, i.e. we propose the use of materials with a temperature-switch behavior (recording the occurrence of a switch) instead of materials with a smooth temperature response. The materials used here are magnetically hard nanoparticles losing order at a transition temperature $T_{\mathrm{C}}$ tunable around room temperature. Nanomagnets with such behavior are usually disregarded for applications including biomedical and biosensing where nanomagnets with a high $T_{\mathrm{C}}\left(>300{ }^{\circ} \mathrm{C}\right)$ are explored. We expect that the logic gates based on these nanomagnets will give valuable insights into temperature in cellular hyperthermia and other demanding applications such as subtle temperature changes during (abnormal) metabolism, heat/lasertriggered release of drugs/genes and ultra-fast PCRs, contributing to their accuracy and development. We further expect that the switching behavior of the nanomagnets developed here can inspire biosensing in general, exploring concepts such as the temperature-selective detection, tagging and separation of "hot" and "cold" analytes.
\end{abstract}

of hyperthermia therapy; ${ }^{1}$ in this case the critical question is, "what is the radiation dose that causes a local temperature overshoot?" The answer to this question can be given by a set/ reset flip-flop logic gate (SR-latch) responding to temperature as one of the inputs, as shown below. A SR-latch changes state as soon as the temperature increases above a well-defined threshold and keeps memory of that state even when the temperature decreases below the threshold. Resetting the original memory state can be performed by a second kind of input. During the last decade, different non-volatile SR-latches based on molecules ${ }^{2}$ and nanoparticles ${ }^{3,4}$ have been developed, mainly exploring photochromic and electrochromic bistable responses. Such gates are able to record information about local $\mathrm{pH}$ and redox conditions, including those occurring in living cells. ${ }^{5}$ Easy integration into biosystems, access to local properties, output detection at a 
distance and no need for external power are major advantages of molecular and nano SR-latch gates and the main driving forces for their development. Hard magnetic nanoparticles display the bistable non-volatile response shown by the SR-latches. A magnetic material is termed "hard" when the direction of its net magnetic moment is difficult to change with an external field, in opposition to "soft" magnetic materials where the net magnetic moment can easily follow the external field. The development of hard magnetic nanoparticles is focused almost exclusively on enhancing and maintaining magnetic hardness up to temperatures well above room temperature. While in contexts such as hard disk recording this is a basic requirement, in a context like biomedical applications, the use of nanoparticles with a dramatic change in magnetic behavior around room temperature represents an unexplored opportunity.

The hard magnetic nanoparticles presented here are iron selenides $\left(\mathrm{Fe}_{3} \mathrm{Se}_{4}\right)$, which have a dramatic change in their magnetic properties at a transition temperature $T_{\mathrm{C}}$ close to room temperature (between $40^{\circ} \mathrm{C}$ and $47^{\circ} \mathrm{C}$ in the bulk form), ${ }^{6,7}$ being hard magnets (i.e. being able to record information) below $T_{\mathrm{C}}$ and losing this ability above this temperature. This behaviour is quite unique and contrasts with the magnetic nanoparticles explored so far (including magnetite, for instance), where the magnetic properties are almost constant around room temperature and $T_{\mathrm{C}}$ is well above room temperature (around $580{ }^{\circ} \mathrm{C}$ in the case of magnetite). Iron selenides are a family of compounds with a wide range of magnetic properties and compositions, including the diamagnetic $\mathrm{FeSe}_{2}$, the ferrimagnetic $\mathrm{Fe}_{3} \mathrm{Se}_{4}$ and $\mathrm{Fe}_{7} \mathrm{Se}_{8}$ and the antiferromagnetic (and superconductor) FeSe. ${ }^{6-8}$ The iron/selenium phase diagram shows that the Se-rich phase $\left(\mathrm{FeSe}_{2}\right)$ and $\mathrm{FeSe}_{2} / \mathrm{Fe}_{3} \mathrm{Se}_{4}$ mixtures are formed at lower temperatures, the phases approaching the 1:1 stoichiometry are formed at higher temperatures, while $\mathrm{Fe}_{3} \mathrm{Se}_{4}$ forms at intermediate temperatures. ${ }^{9}$ With this rich phase diagram, phase purity was the first hurdle to overcome in the design of a successful chemical route for the synthesis of $\mathrm{Fe}_{3} \mathrm{Se}_{4}$ nanocrystals. The current approach to prepare phase pure $\mathrm{Fe}_{3} \mathrm{Se}_{4}$ nanoparticles is based on the thermal decomposition of iron and selenium precursors at intermediate temperatures (300 to $\left.340{ }^{\circ} \mathrm{C}\right),{ }^{10,11}$ where $\mathrm{FeSe}_{2}$ nuclei formed during the heating ramp are transformed into $\mathrm{Fe}_{3} \mathrm{Se}_{4}$. However, without exceptions, this approach leads to nanoparticles and nanostructures with poor control over size, shape and the aggregation state, ${ }^{10-14}$ leading to poor control over the magnetic properties and stability of colloidal dispersions of $\mathrm{Fe}_{3} \mathrm{Se}_{4}$ nanoparticles. This poor control is the present hurdle to overcome.

Our approach to synthesize $\mathrm{Fe}_{3} \mathrm{Se}_{4}$ nanoparticles focused on the use of ligands that could control the reactivity of selenide and iron precursors during the thermal decomposition process, in a strategy to have a low amount of $\mathrm{Fe}^{2+}$ and $\mathrm{Se}^{1-}$ available to react in the (lower) temperature range where $\mathrm{FeSe}_{2}$ can be formed. The best results in terms of phase, size and shape control are obtained when iron oleate and selenium-octadecene are used as cation and anion sources and tetradecylphosphonic acid (TDPA) and 1-dodecanethiol (DDT) as ligands (see the Experimental section and detailed microscopy, diffraction and

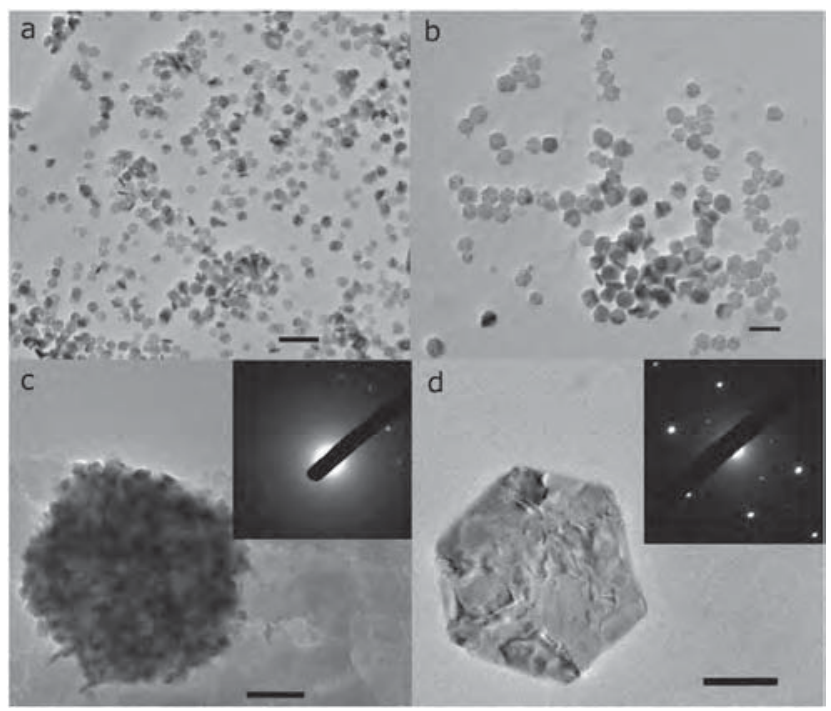

Fig. 1 Electron microscopy images of the $\mathrm{Fe}_{3} \mathrm{Se}_{4}$ nanoplatelets with an average diameter of $250 \mathrm{~nm}$. (a) Low magnification image (scale bar $1 \mu \mathrm{m}$ ); (b) medium magnification image (scale bar $500 \mathrm{~nm}$ ); (c) an $\mathrm{Fe}_{3} \mathrm{Se}_{4}$ nanoplatelet under formation by coherent attachment and an electron diffraction image showing diffraction rings together with spots (scale bar $100 \mathrm{~nm}$ ); and (d) an $\mathrm{Fe}_{3} \mathrm{Se}_{4}$ nanoplatelet and an electron diffraction image showing a [001] zone axis with 6 \{200\} "inner" spots associated with a threefold twinning (scale bar $100 \mathrm{~nm}$ ). Images of nanoplatelets with other average sizes are presented in the ESI. $\dagger$

spectroscopy data comparing nanoplatelets and a reference sample $^{15}$ in the ESI $\left.\dagger\right)$. Within well-defined windows of concentrations, reaction temperatures and ramp slopes, a dramatic improvement in the control over size, shape homogeneity and distribution is obtained, exceeding those of state-of-the-art methods. ${ }^{10-14}$ Within these windows, quasi-hexagonal nanoplatelets with lateral average diameters ranging from 90 to $300 \mathrm{~nm}$ and thicknesses between 40 and $60 \mathrm{~nm}$ are obtained (Fig. 1 and Fig. S1, ESI $\dagger$ ). The key parameters that control the average size of the nanoplatelets are the ramp rate, the structure of iron oleate and its content in free oleic acid. In fact, ironoleate with a larger amount of free oleic acid and unidentate Fe-carboxylate coordination leads to wider nanoplatelets, while purer iron-oleate leads to smaller ones. A relevant influence of these iron-oleate properties was already reported for the case of iron oxide nanoparticles. ${ }^{16}$ The nanoplatelets are obtained at temperatures between $200{ }^{\circ} \mathrm{C}$ and $220{ }^{\circ} \mathrm{C}$, lower than those previously reported $\left(300-340{ }^{\circ} \mathrm{C}\right) .{ }^{10-14}$

Having ensured control over phase, shape, size and size distribution using organic media, phase transfer of the nanoplatelets to aqueous media is the last hurdle before their use in biomedical applications. Our approach focused on the use of a catechol acid, as reported in ref. 17 for the phase transfer of magnetite. Colloidal suspensions of $\mathrm{Fe}_{3} \mathrm{Se}_{4}$ nanoplatelets, stable over a wide $\mathrm{pH}$ range (from 3 to 12 ) in concentrations up to $2 \mathrm{mg} \mathrm{mL} \mathrm{m}^{-1}$, are obtained using caffeic acid as the coating molecule (see the dynamic light scattering analysis of suspensions in Fig. S8, ESI $\dagger$ ). As shown in ref. 17, the carboxylate group of the acid is free to react, allowing further functionalization. 

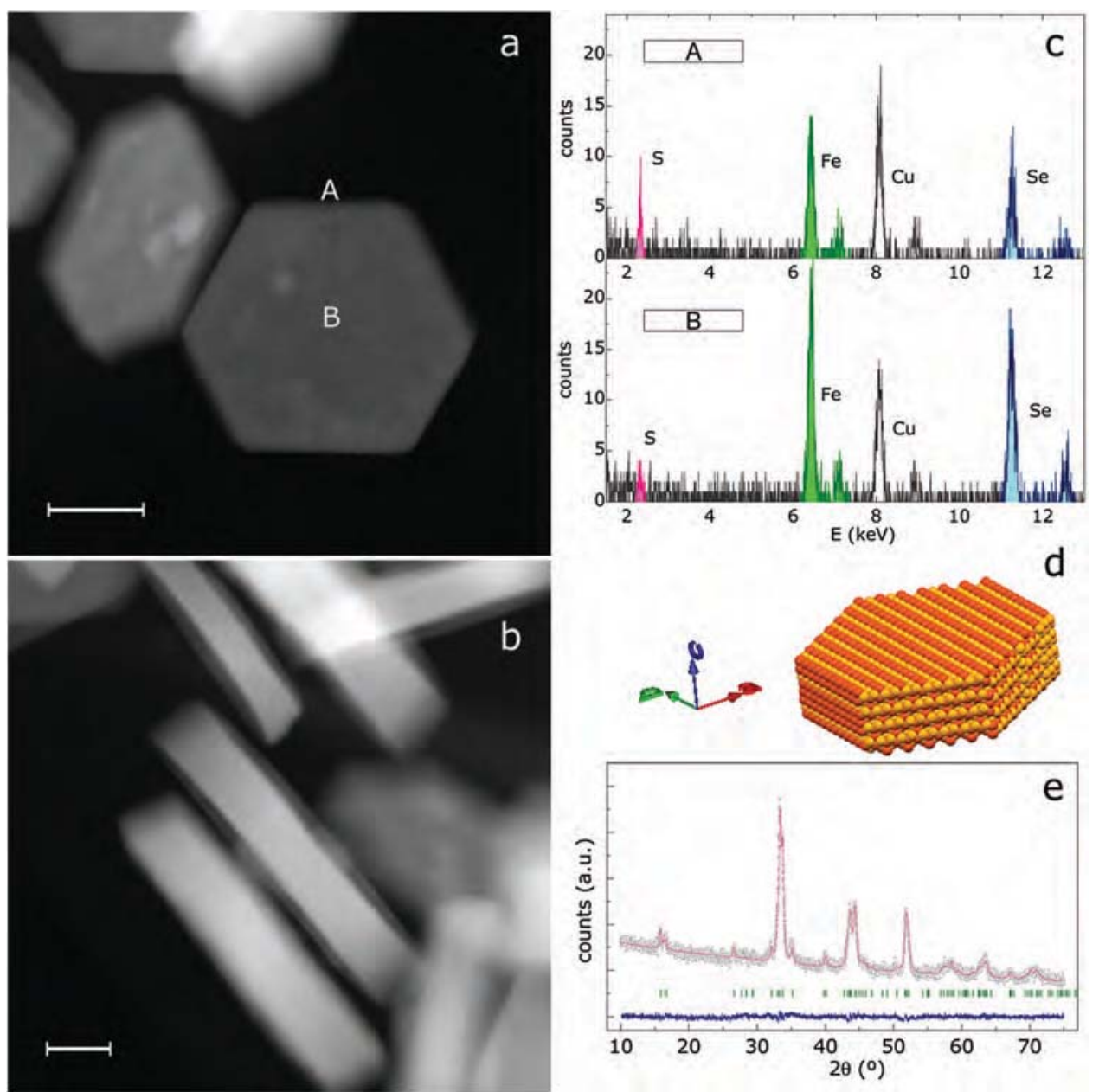

Fig. 2 Electron microscopy images, chemical information and structure of the $\mathrm{Fe}_{3} \mathrm{Se}_{4}$ nanoplatelets with an average diameter of $250 \mathrm{~nm}$. (a and b) Highangle annular dark-field (HAADF) images of $\mathrm{Fe}_{3} \mathrm{Se}_{4}$ nanoplatelets (top-view, scale bar $100 \mathrm{~nm}$; and side-view, scale bar $50 \mathrm{~nm}$ ); (c) EDX spectra at the surface (A) and top (B) of the $\mathrm{Fe}_{3} \mathrm{Se}_{4}$ nanoplatelets; (d) ball-stick representation of an $\mathrm{Fe}_{3} \mathrm{Se}_{4}$ nanoplatelet and (e) X-ray diffraction pattern of the $\mathrm{Fe}_{3} \mathrm{Se}_{4}$ nanoplatelets. The continuous (red) line represents a Rietveld refinement of the XRD data considering space group $P 2 / m$ and cell parameters $a=6.1160(5) \AA, b=3.5025(4) \AA, c=11.144(1) \AA, \beta=91.495(4)^{\circ}$, average apparent (crystallite) size $31 \mathrm{~nm}$ and average maximum strain $12 \%$. Vertical (green) lines represent the positions of the allowed Bragg reflections and continuous (blue, noise-like) lines represent fit residues. XRD data and refinement of nanoplatelets with other average sizes are shown in the ESI. $\dagger$

$\mathrm{Fe}_{3} \mathrm{Se}_{4}$ has a monoclinic quasi-hexagonal crystal structure and the nanoplatelets replicate this structure, growing in thickness along the $c$-axis such that the top and bottom surfaces correspond to the $\{001\}$ family of planes and the lateral surfaces to $\{100\}$ and $\{110\}$ families. Interestingly, all these 3 families generate surfaces composed of "all-Fe" or "all-Se" atoms, while families having mixed $\mathrm{Fe}$ and Se atoms (like $\{010\}$ ) are generally absent as surfaces of the nanoplatelets. This is compatible with a scenario of shape control by preferential surface coordination of the capping ligands. In fact, the nanoplatelets observed on the electron microscope after typical centrifugation and washing techniques are still coated by an organic layer containing sulfur, suggesting the active role of DDT in controlling the formation of the nanoplatelets (Fig. 2). The nanoplatelets present ripples (Fig. 1) and a relatively high degree of strain, as shown by Rietveld refinement to X-ray diffraction (XRD) data (Fig. 2). This high degree of strain is associated with the existence of a 3 -fold twinning observed in the electron diffraction patterns of individual nanoplatelets, where the $(-200)$ and $(200)$ inner spots appear 3 times, rotated by $120^{\circ}$.

During the intermediate states of the synthesis we found proto-nanoplatelets composed of nanocrystals (Fig. 1c). These proto-nanoplatelets display electron diffraction patterns composed of both rings and well-defined spots, suggesting that the growth of the nanoplatelets occurs by the coherent attachment of $\mathrm{Fe}_{3} \mathrm{Se}_{4}$ nanocrystals, being thus particle-mediated rather than atom-mediated. ${ }^{18}$ At the intermediate state shown in Fig. 1c, the nanoplatelet is close to its final size and shape, while the crystallinity is still quite low. The crystallinity, average size and size dispersion evolve until the end of the synthesis procedure. At this end point, chosen to minimize size dispersion, the nanoplatelets are still polycrystalline, being at different stages of crystallization depending on their average sizes. This means that, for each average size, minimizing dispersion leaves the 


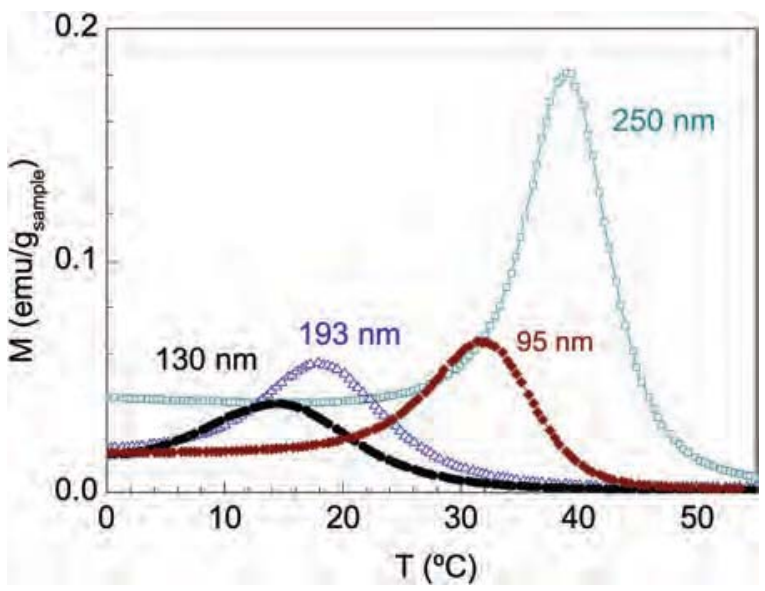

Fig. 3 Magnetization of $\mathrm{Fe}_{3} \mathrm{Se}_{4}$ nanoplatelets with different average lateral diameters (shown in the legend) as a function of temperature. Magnetization was recorded on heating after cooling from $60^{\circ} \mathrm{C}$ down to $0{ }^{\circ} \mathrm{C}$ in the absence of an external field. $T_{C}$ is the temperature where the derivative of magnetization is minimum, i.e., slightly above the maximum. $T_{C}$ increases monotonically with the increase of the crystallite size and not with the increase of the diameter of the nanoplatelets.

nanoplatelets at different degrees of crystallization, such that the relation between size and crystallite size is not monotonic (see Table S1, ESI $\dagger$ ).

While in the micro- and nano-particles reported so far $T_{\mathrm{C}}$ is between $47{ }^{\circ} \mathrm{C}$ and $67{ }^{\circ} \mathrm{C},{ }^{10-14}$ the nanoplatelets reported here have a $T_{\mathrm{C}}$ tunable from $18{ }^{\circ} \mathrm{C}$ to $42{ }^{\circ} \mathrm{C}$, increasing with the increase of the crystallite size determined by XRD (Fig. 3, and Fig. S3 and S5, ESI $\dagger$ ), as usually found in finite-size systems. ${ }^{19}$ Below $T_{\mathrm{C}}$, the nanoplatelets behave as hard ferrimagnets presenting a spontaneous (remanent) magnetization in the absence of an external field $\left(M_{\mathrm{r}}\right.$, Fig. S7, ESI $\left.\dagger\right)$. The coercive field $\left(H_{\mathrm{C}}\right.$, Fig. S7, ESI $\left.\dagger\right)$ corresponds to the field needed to bring this remanent magnetization down to zero. As the temperature decreases across $T_{\mathrm{C}}, H_{\mathrm{C}}$ has a pronounced increase up to values of the order of $4 \mathrm{kOe}$, which is high enough to prevent demagnetization under normal conditions and small enough to be overcome using a strong permanent magnet.

Hard magnetic nanocrystals with $T_{\mathrm{C}}$ close to room temperature, like the $\mathrm{Fe}_{3} \mathrm{Se}_{4}$ nanoplatelets presented here, are useless in traditional magnetic recording, where temperature-sensitive recording is a problem. However, this sensitivity can give valuable insight into materials and systems which are sensitive in the same temperature range, such as tissues, cells, biomolecules and soft materials in general. For instance, $\mathrm{Fe}_{3} \mathrm{Se}_{4}$ nanoplatelets with a $T_{\mathrm{C}}$ close to $42{ }^{\circ} \mathrm{C}$ can record information of an event of temperature increase above the normal cell temperature. Below $T_{\mathrm{C}}$, i.e. in the normal temperature range, the nanoplatelets may have a stable high or a stable low value of $M_{\mathrm{r}}$ (with the difference between both being at least an order of magnitude), depending on which stimuli was applied last: a magnetic field or a temperature above $T_{\mathrm{C}}$, respectively (Fig. 4 and Fig. S9 and S15, ESI, $\dagger$ for further details). After setting the $\mathrm{Fe}_{3} \mathrm{Se}_{4}$ nanoplatelets into a high state by the application of a magnetic field, a temperature increase above the threshold defined by $T_{\mathrm{C}}$ will reset $M_{\mathrm{r}}$ into a low state, thus recording information about that increase. After reading this information, the nanoplatelets can be set again in a state of high $M_{\mathrm{r}}$, which can be easily performed with an external magnetic field. This corresponds to a SR-latch where temperature and the field are the input stimuli and (remanent) magnetization is the output, with this gate being able to answer the question "did the temperature exceed the threshold defined by $T_{\mathrm{C}}$ since the last time an external field was applied?". In the context of cells, this question arises, for instance, after the application of targeted hyperthermia, where heat sources placed close to or inside cells are triggered at distance. SR-latches are basic memory elements with one output having two possible stable states (high and low) and two inputs: one having the role of setting the output high and the other having the role of setting the output low. In electronics, inputs are voltage levels connected by two NOR gates to one output and its negation (see Fig. S9, ESI $\dagger$ ). In the present implementation of a SR-latch using magnetic nanoplatelets, the output is magnetization, which can be set high using a magnetic field as an input and reset low using temperature as the second input. Since the nanoplatelets are hard magnets, high and low magnetization states remain stable without energy consumption until the temperature (or field) is changed, responding to temperature in the time-frame of the spin-lattice relaxation (typically between $10^{-7}$ and $10^{-4} \mathrm{~s}$ ). ${ }^{20}$

In order to illustrate the use of the $\mathrm{Fe}_{3} \mathrm{Se}_{4}$ nanoplatelets as SR-latches in cells during hyperthermia, we used a layer of nanoplatelets with $T_{\mathrm{C}}=42{ }^{\circ} \mathrm{C}$ embedded in a layer of Matrige ${ }^{\mathrm{TM}}$ where prostate cancer cells were seeded (see Fig. 5 schematics and details in the ESI $\dagger$ ). The layered system was designed to keep the expected maximum temperature drop between the nanoplatelets and cells below $\sim 0.5{ }^{\circ} \mathrm{C}$ in a compromise between an accurate temperature assessment and being as less intrusive as possible (see Fig. S10, ESI, $\dagger$ for details). The layered system was then heated at a distance using a near infrared (NIR - $850 \mathrm{~nm}$ ) LED. In this setup, the nanoplatelets act as both logic gates and the main NIR light absorbers, i.e., the main heat sources.

The temperature increase was manipulated by changing the intensity of the LED and the irradiation time. For a total irradiation time of $5 \mathrm{~min}$, the logic gates indicated that the temperature threshold of $42{ }^{\circ} \mathrm{C}$ is surpassed at a power density between 456 and $570 \mathrm{~mW} \mathrm{~cm}{ }^{-2}$ (see the ESI, $\dagger$ Fig. S11). This result shows a good correlation with the binary indication given by the live/dead assay: in the non-irradiated control and for LED powers below the threshold determined by the logic gates (Fig. 5B1-B6), the assay shows that the great majority of cells are viable $24 \mathrm{~h}$ after irradiation, while above that power threshold the opposite is observed (Fig. 5B7-B9). These results highlight the use of the $\mathrm{Fe}_{3} \mathrm{Se}_{4}$ nanoplatelets to simultaneously gain insight as a quality control technology for temperature thresholds during treatments, while acting as NIR light absorbers in anti-cancer hyperthermia.

The nanoplatelets reported here represent a landmark in the size control of $\mathrm{Fe}_{3} \mathrm{Se}_{4}$ nanoparticles, leading to good control over their magnetic properties and stability in water. Such control of properties opens the way to new applications exploring the unique characteristics of $\mathrm{Fe}_{3} \mathrm{Se}_{4}$ nanoparticles: ability to 


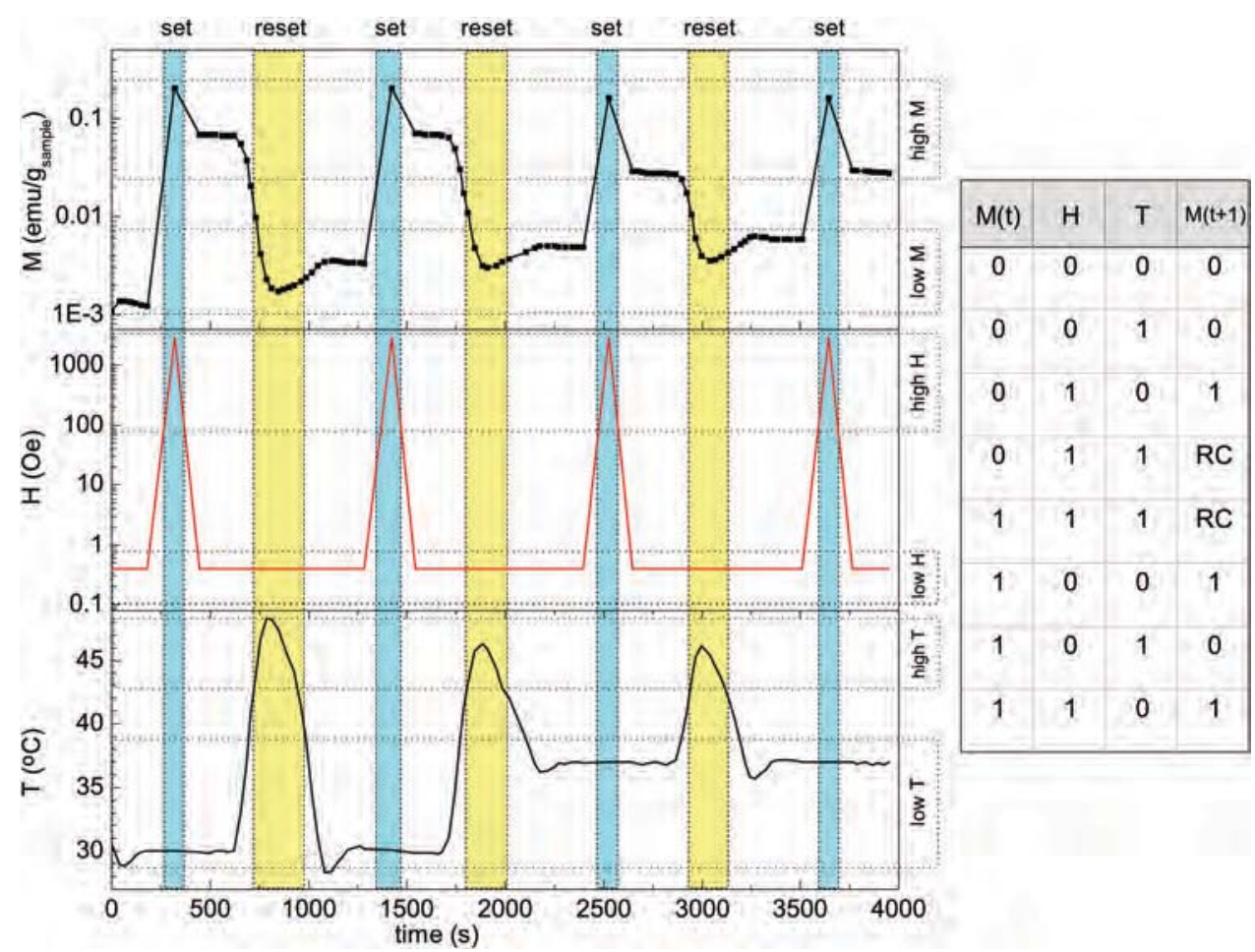

Fig. 4 Example of the time diagram of the bistable gate. Basic operations of the gate based on the change in the magnetization $M$ (output) response of the $\mathrm{Fe}_{3} \mathrm{Se}_{4}$ nanoplatelets (average diameter $250 \mathrm{~nm}$ ) to the field $H$ (set) and temperature $T$ (reset) inputs. Time diagrams obtained for the remaining samples are shown in Fig. S9 (ESI†). Stability tests on field temperature cycling and the stability of the magnetization response over time are shown in Fig. S18 and S19 (ESI $\dagger$ ). For a given initial output sate $M(t)$ (which can be high 1 or low 0 ), the states of the two inputs $H$ and $T$ will determine the final state $M(t+1)$ as shown in the table. If both $H$ and $T$ are high, the final state $M(t+1)$ is ill-defined and will depend on which $H$ or $T$ turns back to a low state first, in a so-called race condition (RC).

record information and inability to keep that information above a critical temperature $T_{\mathrm{C}}$ tunable in a range around room temperature. In other words, $\mathrm{Fe}_{3} \mathrm{Se}_{4}$ nanoparticles are not suitable for long-term data storage but are valuable for monitoring materials having also some sort of critical change in their behaviour at a temperature close to $T_{\mathrm{C}}$, such as tissues, cells, biomolecules and soft matter in general. A noteworthy example is the combination of $\mathrm{Fe}_{3} \mathrm{Se}_{4}$ nanomagnets and cells sharing a critical temperature, with this combination being explored to keep track of threshold temperatures in the context of hyperthermia, as shown here. This proof of concept opens the pathway to other noteworthy applications such as monitoring temperature thresholds in heat-triggered release of drugs or genetic therapy and monitoring temperature thresholds in ultrafast local increase of temperature in polymerase chain reaction. ${ }^{21}$ In addition, the ultrafast response to temperature and the nanometer size of the $\mathrm{Fe}_{3} \mathrm{Se}_{4}$ platelets allow them to be further explored in limit situations where both high temporal and high space resolution are required, avoiding the typical constraints of nanoscale thermometers. ${ }^{22}$

\section{Experimental}

$\mathrm{Fe}_{3} \mathrm{Se}_{4}$ nanoplatelets were synthesized by the thermal decomposition of $\mathrm{Fe}$-oleate in the presence of a Se-octadecene complex, tetradecylphosphonic acid (TDPA) and 1-dodecanethiol (DDT). Typical amounts used were $210 \mathrm{mg}$ of Fe-oleate, $10 \mathrm{~g}$ of Se-octadecene, $150 \mathrm{mg}$ of DDT and $30 \mathrm{mg}$ of TDPA. The mixture was allowed to purge at low pressure and $120{ }^{\circ} \mathrm{C}$ for $30 \mathrm{~min}$, and ramped at $5{ }^{\circ} \mathrm{C} \min ^{-1}$ up to $210{ }^{\circ} \mathrm{C}$, where it remained for $1 \mathrm{~h}$. Fe-oleate was prepared and washed using standard procedures. ${ }^{16}$ The Se-octadecene complex was also prepared using standard procedures ${ }^{23}$ by the reaction of $434 \mathrm{mg}$ of metallic Se powder with $75.3 \mathrm{~g}$ of 1-octadecene for $30 \mathrm{~min}$ at $180{ }^{\circ} \mathrm{C}$.

Phase transfer of the $\mathrm{Fe}_{3} \mathrm{Se}_{4}$ nanoplatelets was performed by exchanging the DDT/TDPA molecules on the nanoplatelets' surfaces for molecules of caffeic acid. $10 \mathrm{mg}$ of nanoplatelets were dispersed in THF, along with $50 \mathrm{mg}$ of caffeic acid. The mixture was left at $50{ }^{\circ} \mathrm{C}$ for $4 \mathrm{~h}$ under stirring to promote the exchange of molecules. $100 \mu \mathrm{L}$ of a $0.5 \mathrm{M}$ solution of $\mathrm{NaOH}$ were added to the mixtures and the particles were centrifuged and separated from the THF, which were dispersed in about $2 \mathrm{~mL}$ of distilled water without further washing.

A water suspension of $\mathrm{Fe}_{3} \mathrm{Se}_{4}$ nanoplatelets was cast in $\mu$-slide angiogenesis Ibidi imaging chambers until a uniform layer was formed. Matrigel ${ }^{\mathrm{TM}}$ was then deposited and allowed to crosslink with the nanoplatelets at $37{ }^{\circ} \mathrm{C}$ for $2 \mathrm{~h}$. This dual deposition strategy allowed for the formation of a cell adhesive substrate for PC-3 cell proliferation. Prostate cancer cells were then seeded at a density of $1.3 \times 10^{5}$ cells per $\mathrm{cm}^{2}$ and allowed to proliferate for $24 \mathrm{~h}$. 

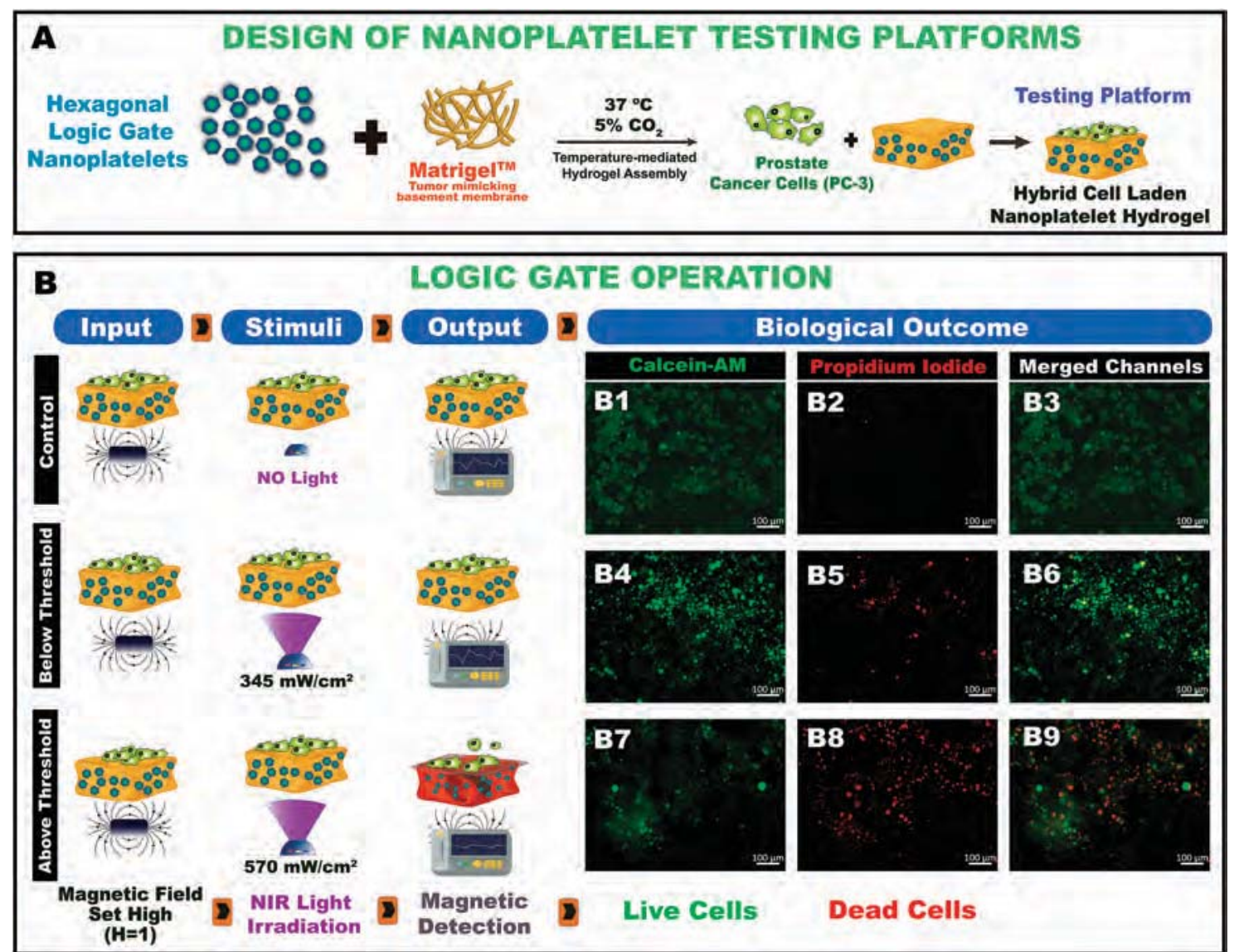

Fig. 5 (A) Scheme showing the assembly of the nanoplatelets with the Matrigel ${ }^{\mathrm{TM}}$ and Cells, giving rise to the testing platform for logic gate nanoplatelet operation under temperature and field stimuli. (B) Scheme showing the use of the logic gates to record a possible surpassing of the $42{ }^{\circ} \mathrm{C}$ threshold temperature during irradiation and fluorescence microscopy micrographs of irradiated PC-3 cells: first, the nanoplatelets are set in a high state; second, the cells are irradiated with different NIR doses; third, the state of the nanoplatelets is evaluated after irradiation. The state of the cells is evaluated after $24 \mathrm{~h}$ using a live-dead assay: (B1 to B3) non-irradiated PC-3 cells, control; (B4 to B6) NIR irradiated PC-3 cells with the recorded temperature below the set threshold ( $345 \mathrm{~mW} \mathrm{~cm}^{-2}, 5 \mathrm{~min}$ ); (B7 to B9) NIR irradiated PC-3 cells with the temperature above the threshold (570 $\left.\mathrm{mW}_{\mathrm{cm}}^{-2}, 5 \mathrm{~min}\right)$. Green channel: calcein-AM, red-channel: propidium iodide.

Before irradiation, the $\mathrm{Fe}_{3} \mathrm{Se}_{4}$ nanoplatelets were magnetized (i.e. set in a high state $M=1$, by applying a high field $H=1$ ) using a cylindrical neodymium-iron-boron magnet (diameter $8 \mathrm{~mm}$, height $30 \mathrm{~mm}$, N42). Irradiation was then performed using an 850 NIR LED (Oslon PowerStar) with power densities between 285 and $570 \mathrm{~mW} \mathrm{~cm}^{-2}$ (according to specifications) for $5 \mathrm{~min}$. This irradiation may lead (or not) to a change in temperature state from low to high and it may thus change (or not) the magnetization state from high to low. This possible change in the magnetization state after the irradiation procedure was measured using a fluxgate magnetometer (FL1-100 Stefan Mayer Instruments) embedded in a permalloy foil to decrease the ambient magnetic field and noise at the fluxgate. At last, on the following day, cells were incubated with Calcein-AM/PI for $30 \mathrm{~min}$ at $37^{\circ} \mathrm{C}$. The cells were then washed 3 times with PBS and imaged using a Zeiss Imager M2 fluorescence microscope.

\section{Conflicts of interest}

There are no conflicts to declare.

\section{Acknowledgements}

We thank Manuel Martins for fruitful discussions during the early stages of this work. We acknowledge J. Lindén for kindly providing us with the reference $\mathrm{Fe}_{3} \mathrm{Se}_{4}$ sample and Guillermo Antorrena for the XPS measurements. We also acknowledge Tera Analysis and Vladimir Podnos for kindly providing us with a professional version of QuickField ${ }^{\mathrm{TM}}$. This work was developed within the scope of the projects CoolPoint P2020-PTDC-CTMNAN-4511-2014 and CICECO-Aveiro Institute of Materials, POCI-01-0145-FEDER-007679 (FCT Ref. UID/CTM/50011/2013), financed by national funds through the FCT/MEC and co-financed by FEDER under the PT2020 Partnership Agreement. The work in Zaragoza was supported by the research grant MAT2014-54975-R from the Spanish Ministry of Science and Innovation. Access to advanced electron microscopy facilities received funding from the European Union Seventh Framework Programme under Grant Agreement 312483 - ESTEEM2 (Integrated Infrastructure Initiative I3). This work was also supported by a STSM Grant from COST Action MP1306 and TD1402. RPOS, VMG, FLS and 
NJOS acknowledge FCT for the PD/BD/116850/2016, SFRH/BPD/ 119983/2016, IF/00222/2015 and IF/01533/2015 grants.

\section{References}

1 R. T. Gordon, J. R. Hines and D. Gordon, Med. Hypotheses, 1979, 5, 83.

2 A. de Silva, Molecular Logic-based Computation, Monographs in Supramolecular Chemistry, Royal Society of Chemistry, 2016, ISBN 9781782626237, https://books.google.pt/ books?id=3msoDwAAQBAJ.

3 I. Medalsy, M. Klein, A. Heyman, O. Shoseyov, F. Remacle, R. D. Levine and D. Porath, Nat. Nanotechnol., 2010, 5, 451, DOI: $10.1038 /$ nnano.2010.62.

4 L. X. Mu, W. S. Shi, T. P. Zhang, H. Y. Zhang, Y. Wang, G. W. She, Y. H. Gao, P. F. Wang, J. C. Chang and S. T. Lee, Appl. Phys. Lett., 2011, 98, 163101, DOI: 10.1063/1.3581884.

5 E. W. Miller, S. X. Bian and C. J. Chang, J. Am. Chem. Soc., 2007, 129, 3458, DOI: 10.1021/ja0668973, pMID: 17335279.

6 T. Hirone and S. Chiba, J. Phys. Soc. Jpn., 1956, 11, 666, DOI: 10.1143/JPSJ.11.666.

7 P. Terzieff and K. L. Komarek, Monatsh. Chem., 1978, 109, 1037, DOI: 10.1007/BF00913006, ISSN 1434-4475.

8 J. E. Dutrizac, M. B. I. Janjua and J. M. Toguri, Can. J. Chem., 1968, 46, 1171, DOI: 10.1139/v68-200.

9 W. Schuster, H. Mikler and K. L. Komarek, Monatsh. Chem., 1979, 110, 1153, DOI: 10.1007/BF00910963, ISSN 1434-4475.

10 H. Zhang, G. Long, D. Li, R. Sabirianov and H. Zeng, Chem. Mater., 2011, 23, 3769, DOI: 10.1021/cm201610k.

11 I. S. Lyubutin, C.-R. Lin, K. O. Funtov, T. V. Dmitrieva, S. S. Starchikov, Y.-J. Siao and M.-L. Chen, J. Chem. Phys., 2014, 141, 044704, http://scitation.aip.org/content/aip/journal/ jcp/141/4/10.1063/1.4887356.
12 S. J. Li, D. Li, J. J. Jiang, G. B. Liu, S. Ma, W. Liu and Z. D. Zhang, J. Appl. Phys., 2014, 115, 17.

13 M. Sen Bishwas, R. Das and P. Poddar, J. Phys. Chem. C, 2014, 118, 4016, DOI: 10.1021/jp411956q.

14 S.-J. Li, D. Li, W. Liu and Z. Zhang, Nanoscale, 2015, 7, 5395, DOI: 10.1039/C4NR07287A.

15 R. Pohjonen, O. Mustonen, M. Karppinen and J. Lindén, J. Alloys Compd., 2018, 746, 135, ISSN 0925-8388, http://www. sciencedirect.com/science/article/pii/S0925838818307461.

16 L. M. Bronstein, X. Huang, J. Retrum, A. Schmucker, M. Pink, B. D. Stein and B. Dragnea, Chem. Mater., 2007, 19, 3624 .

17 Y. Liu, T. Chen, C. Wu, L. Qiu, R. Hu, J. Li, S. Cansiz, L. Zhang, C. Cui and G. Zhu, et al., J. Am. Chem. Soc., 2014, 136, 12552, DOI: 10.1021/ja5060324, pMID: 25140614.

18 H. You and J. Fang, Nano Today, 2016, 11, 145, ISSN 17480132, http://www.sciencedirect.com/science/article/pii/S17480 13215300888.

19 M. E. Fisher and M. N. Barber, Phys. Rev. Lett., 1972, 28, 1516.

20 E. Abrahams, Advances in Electronics and Electron Physics, Academic Press, 1954, vol. 6, pp. 47-68, http://www.science direct.com/science/article/pii/S0065253908601316.

21 J.-H. Lee, Z. Cheglakov, J. Yi, T. M. Cronin, K. J. Gibson, B. Tian and Y. Weizmann, J. Am. Chem. Soc., 2017, 139, 8054, DOI: 10.1021/jacs.7b01779, pMID: 28457135.

22 C. D. S. Brites, P. P. Lima, N. J. O. Silva, A. Millan, V. S. Amaral, F. Palacio and L. D. Carlos, Nanoscale, 2012, 4, 4799, DOI: 10.1039/C2NR30663H.

23 C. Bullen, J. van Embden, J. Jasieniak, J. E. Cosgriff, R. J. Mulder, E. Rizzardo, M. Gu and C. L. Raston, Chem. Mater., 2010, 22, 4135, DOI: 10.1021/cm903813r. 